\title{
1 A versatile high throughput strategy for cloning the env gene of HIV-1
}

2 Nitesh Mishra ${ }^{1}$, Ayushman Dobhal $^{1,2}$, Shaifali Sharma ${ }^{1,2}$, Kalpana Luthra $^{1, \#}$.

$3 \quad{ }^{1}$ Department of Biochemistry, All India Institute of Medical Sciences, New Delhi, India - 110029.

$4 \quad{ }^{2}$ These authors contributed equally.

$5 \quad$ "corresponding author (kalpanaluthra@gmail.com)

\section{Abstract}

7 The trimeric envelope glycoprotein (gp120/gp41) $)_{3}$ of human immunodeficiency virus-1 (HIV-1)

8 mediates viral and host cell membrane fusion, initiated by binding of viral envelope gp120 protein to

9 the CD4 receptor on host immune cells. Functional env genes from infected individuals have been

10 widely used as templates for vaccine design, for setting up viral neutralization assays and to study the

11 viral evolution and pathogenesis. Traditional topoisomerase or T4 DNA polymerase mediated

12 approaches for cloning single genome amplified (SGA) env genes are labor-intensive, cost-ineffective

13 with low-throughput, thereby enabling functional analysis of only a limited number of env genes from

14 the diverse circulating quasispecies in infected individuals. Herein, we report an efficient, easy to

15 optimize and high-throughput approach for cloning diverse HIV-1 env genes. Multiple env/rev gene

16 cassettes, derived from infected infants, were subjected to SGA using Phusion polymerase and

17 utilized as megaprimers in overlap extension PCR mediated cloning (OEC), circumventing the

18 requirement for novel enzymes. Furthermore, utilization of Phusion polymerase for both the

19 amplification of env/rev cassettes and OEC allows convenient monitoring and optimization, thereby

20 providing much greater flexibility and versatility for analysis of env genes from HIV-1 infected

21 individuals.

\section{Introduction}

23 Rapid evolution of HIV-1 strains is increasing the global viral diversity. High baseline rates of mutation

24 (nucleotide substitution rate) due to an error prone reverse transcriptase, rapid replication cycle, in

25 combination with continuous immune-driven selection within the host generates a unique and complex viral quasispecies in each infected individual $(1,2)$. In the context of HIV-1, the viral species in each infected individual accrues immunologically relevant mutations, generating a viral pool of highly complex and unique variants circulating within each host (3-5). Population based consensus viral sequences are used to define wildtype HIV-1 virus, but given the extensive diversity, each variant within a clade is distant from the consensus sequence, giving rise to millions of coexisting variants in circulation. Developing an HIV-1 vaccine is a global priority, and the discovery of broadly neutralizing antibodies (bnAbs) has invigorated the field of HIV-1 vaccine research $(6,7)$. bnAbs target the envelope glycoprotein (env) of HIV-1 within defined epitopes, though inferred germlines (iGLs) of several bnAbs do not bind to most of the difficult-to-neutralize HIV-1 isolate. Identification and/or engineering of env variants capable of engaging and shepherding bnAb development pathway are a key focus of lineage-based vaccine approach (8-12). 
37 Functional characterization of env gene provides key information for vaccine design, identify env

38 candidates capable of serving as immunogens, understanding viral evolution and pathogenesis in

39 response to host response, or mutational landscape among several other virological and

40 immunological functions $(3-5,8,13,14)$. Traditionally, envelopes are characterized by generating

41 pseudoviruses (env genes from different hosts cloned in mammalian expression vectors) in

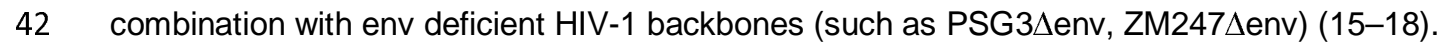

43 Conventional approaches for amplifying env gene via single genome amplification (SGA) and cloning

44 env gene are time and labor intensive processes. In addition, utilization of topoisomerases (TOPO

45 cloning) or T4 DNA polymerases (SLIC, or Infusion cloning) to clone env gene can only be done at

46 low throughout as increasing cost associated with cloning multiple env genes restricts the cloning to

47 few select env clones $(15,19,20)$. Single env clones are not representative of the diversity of HIV-1 in

48 a patient's blood sample (19,21-25). The SGA technique using traditional TOPO or Infusion cloning

49 kits to generate functional env clones that represent majority of the circulating quasispecies is low-

50 throughput and not cost-effective due to the involvement of multiple PCR reactions, purification steps

51 and ligation reactions.

52 In the age of high-throughput molecular and systems biology, several approaches have been

53 described to overcome the limitations associated with conventional cloning approaches (26-41).

54 Genes are often identified as difficult-to-clone that exhibit features such as uneven base distribution,

55 secondary structures, toxicity to bacterial strain used for cloning and the HIV-1 env gene exhibits all

56 of these features (42-44). Few select strategies exist by which the env gene can be cloned with high

57 efficiency. One such approach called overlap extension cloning (OEC) involves solely a a PCR-

58 based cloning workflow that offers highest versatility in terms of optimizing cloning approaches (26-

59 28). In OEC, the insert of interest (called a megaprimer) is used in conjunction with a proofreading

60 polymerase that does not have strand displacement activity (such as Phusion polymerase) to swap

61 the insert into vector backbone, and is sequence and ligation independent. Variants of OEC like IOEP

62 (Improved Overlap Extension PCR) (29), IVA (In Vivo Assembly) (39), AQUA (Advanced QUick

63 Assembly) (40), CPEC (Circular Polymerase Extension Cloning) (41) have initiated new avenues for

64 high-throughput cloning. Most of these approaches work well for subcloning (swapping inserts from

65 one plasmid backbone into another) but fail when applied to cloning gene of interest from biological

66 samples. This study aimed to design a cost-effective and high-throughput approach to clone HIV-1

67 env gene in order to capture maximum functional diversity of the circulating viral variants in infected

68 individuals. A low-cost, easy to optimize, and high-throughput method, based on the principles of

69 overlap extension cloning, to construct env clones representing the functional diversity of circulating

70 HIV-1 viral variants is proposed herein, allowing rapid production of heterogenous patient derived env

71 genes. The robustness of this strategy was further evaluated and confirmed by cloning viral env gene

72 from eight HIV-1 infected infants.

\section{Results and Discussion}

74 Overlap extension cloning (Fig. 1) is a PCR-based cloning technique and therefore primer design is

75 critical for a successful OEC, as non-specific primer binding can lead to amplification of spurious PCR 
products. Conserved regions of the HIV-1 envelope were selected for primers design to clone the viral env gene, to accommodate for the high envelope sequence diversity, uneven base composition (on average $36 \%$ adenine, $22 \%$ thymine, $24 \%$ guanine and $18 \%$ cytosine) and high propensity of secondary structure in the AT-rich HIV-1 genome $(42,43)$. Given that HIV-1 diverges overtime $(1,2)$, we first updated our primer repository to ensure optimum amplification of the env gene (onwards called env/rev cassettes, as partial fragment for rev gene is part of the env gene). Two-step nested PCR reactions were used to amplify env/rev cassettes, where the forward primers (Fw1 and Fw2) bind to the tat region (upstream to the env gene), and the reverse primers (Rv1 and Rv2) bind to nef region (downstream to the env gene) (Fig. 2a). Our major goal was to design a cloning strategy where env/rev cassettes can be cloned in a single tube without the need for novel enzymes like topoisomerases, exonucleases, T4 DNA polymerases, Taq DNA ligase or recombinases. Therefore, the robustness of the nested PCR for env/rev amplification was of utmost importance. Using the parameters of Clade $\mathrm{C}$ tat and nef sequences of Indian origin available in Los Alamos National Laboratory's HIV Database, we selected a total of 276 tat (HXB2 numbering $5831-6045$ ) and 140 nef (HXB2 numbering 8797 - 9417) sequences. Sequences were aligned with HIValign tool using the HMM-align model with compensating mutation occurring within 5 codons to compensate frameshift and using previously reported primers (15-17) as anchors [VIF1 as Fw1 primer (HXB2 numbering 5852 - 5876), ENVA as Fw2 primer (HXB2 numbering 5951 - 5980), OFM19 as Rv1 primer (HXB2 numbering 9604 - 9632) and ENVN as Rv2 primer (HXB2 numbering 9144 -9172)] (Fig. 2a). A total of 4 Fw1, 2 Rv1, 12 Fw2 and 6 Rv2 primers were selected (Table 1) to address the diversity observed within the geographically restricted sequences of Indian origin (Fig. $\mathbf{2 b}-\mathbf{c}$ ). We first utilized a multiplex PCR approach where all Fw1 and Rv1 primers were pooled for the first round, and all Fw2 and Rv2 primers were pooled for the amplification via nested PCR. Though consistent amplification of env/rev cassettes across samples was achieved, the efficiency of PCR was low and in addition smearing and/or non-specific amplification were notably observed (Fig. 2d). To overcome this, we next performed nested PCR using all of the possible primer pair combinations (a total of 576 individual PCR reactions), Four of these primer pairs yielded a prominent env/rev amplification from 8 HIV-1 infected samples (Fig. 2e), randomly selected from a recent cohort of HIV-1 infected infants (45). Despite employing optimized primers selected from an exhaustive panel, the cloning efficiency of the env/rev cassettes via SGA amplification varied between samples. Further optimization of PCR for amplifying env/rev cassettes was done by varying the concentration of existing reactants $s$ (magnesium, DMSO, BSA, PEG8000, primers). In addition, reducing the number of cycles in the first round of nested PCR significantly reduced spurious, non-specific products observed in certain samples.

To generate overlaps (plasmid sequences at both ends) that can be used for OEC, we added overhangs of 15,25 and $35 \mathrm{bp}$ randomly selected, upstream of the multiple cloning site (MCS) region of pcDNA3.1, to the chimeric primers generated by combining four primers, with 5 end complementary to the vector and 3 ' ends optimum for amplification of env/rev cassettes. Interestingly, none of the chimeric primer pair combination could amplify env/rev cassettes from the eight patient samples but prominent amplification was seen when plasmids containing env/rev cassettes were 
116 used. Weak amplification with $15 \mathrm{bp}$ overhang chimeric primers were however observed in two of the eight samples (data not shown). HIV-1 contains an above average percentage of adenine (A) nucleotides, while cysteine (C) nucleotides are extremely low (Supplementary Fig. 1). Designing primers with standard primer designing rules to accommodate high HIV-1 diversity is therefore difficult. Primers used for amplification of env/rev cassettes are typically AT-rich while the overhang sequences taken from plasmids show typical pattern of base distribution (optimally dispersed pyrimidine and purine patterns). Addition of plasmid sequences to primers optimized for HIV-1 env/rev amplification, therefore, generates suboptimal chimeric primers (uneven distribution of pyrimidine and purine bases) plausibly explaining the failure of chimeric primers to amplify env/rev cassettes from biological samples. As amplification of env/rev cassettes with chimeric primers gave unsatisfactory results, we utilized an alternate approach for performing OEC with env/rev cassettes serving as megaprimers. We placed the Fw2 and Rv2 sequences 150 bp apart around the multiple cloning site (MCS) of pcDNA3.1 (pcDNA3.1_ITR), generating a plasmid that can be directly used for OEC using env/rev cassettes amplified from patient samples (Supplementary Fig. 2).

OEC has been reported to use linear amplification, typically generating amplicons too little to be visualized on agarose gels. In addition, the effectiveness of OEC has been shown to be dependent upon megaprimer concentration, with the megaprimer concentration being inversely proportional to size (26). To obtain good yield, four parameters were sequentially optimized, namely the number of cycles, annealing temperatures, concentration of recipient plasmid (pcDNA3.1_ITR), and concentration of megaprimer (env/rev cassette). We began our iterative optimization of OEC using 10 to $100 \mathrm{ng}$ of pcDNA3.1_ITR and PCR purified env/rev cassettes at excess ratios of 1:3 to 1:300 in 25$\mu \mathrm{l}$ reactions using previously reported reaction conditions. The PCR reaction was inhibited at high DNA concentrations (100 ng of pcDNA3.1_ITR and $1745 \mathrm{ng}$ of PCR purified env/rev cassettes at 1:35 molar excess ratios) (Fig. 3a). High concentrations of insert and lower annealing temperature for megaprimers have been implicated as necessary for successful OEC $(26,27)$, however we found that a high concentration of the env gene insert often led to failed PCR. A molar excess ratio of 1:5 to 1:10 of env/rev cassettes to $50 \mathrm{ng}$ of pcDNA3.1_ITR gave the most optimum results, though multiple bands after OEC were observed, when the vector to insert ratio exceeded 1:25 (data not shown). Higher annealing temperatures were required to maximize OEC efficiency (Fig. 3b), plausibly by minimizing the formation of interfering secondary structures, typical for lentiviruses $(42,43)$, as hairpin structures within the template region can hinder the PCR amplification. Addition of BSA and removal of DMSO serendipitously eliminated the non-specific multiple bands observed with OEC. As DMSO disrupts secondary structure formation, the observed decrease in spurious PCR amplification in its absence is counterintuitive, and requires further exploration. In addition, the high concentration of pcDNA3.1_ITR (10 ng) made Dpnl digestion necessary in the OEC workflow, though Dpnl could be added straight to Phusion HF buffer, thereby bypassing the need for purification steps. These reaction conditions were then taken forward for further optimization.

The number of cycles in OEC have been inversely linked to cloning efficiency and therefore to optimize the cycle number for amplification, we next performed OEC in increment of 2 cycles, with a 
minimum amplification of 10 cycles to begin with. Considerable variation was observed for OEC efficiency as a measure of PCR cycles between samples (cfu/ng of DNA, figure), though 14-16 cycles gave most satisfactory results (Fig. 4a). Few empty colonies were also observed in OEC done with pcDNA3.1_ITR alone (serving as the vector control), presumably due to the carry-over of the vector, as Dpnl digestion was performed directly in PCR buffer. Though commercially available restriction enzymes are active in PCR buffers, reaction efficiency varies between samples. To address the unwanted vector carryover, we subcloned CCDB gene (46) (from vector pZM247Fv1 $\triangle E n v$, procured from NIH AIDS Reagent Program) in-frame to T7 Promoter and between the Fw2 and Rv2 sites of pcDNA3.1_ITR via OEC. Utilization of the suicide vector (pcDNA3.1_ITR_CCDB) containing CCDB gene for OEC of env/rev cassettes completely removed unwanted vector carry-over as well as the requirement for Dpnl digestion.

Considering our initial goal was to develop a system that can be globally implemented across labs working with HIV-1, we reasoned using readily available pseudoviral env clones used across labs for standardized assessment of bnAbs or vaccine-induced sera nAbs as vectors for OEC. Herein, we first mutated the ENVA and ENVN sites in 329_14_B1 backbone (a subtype C pseudoviral clone, accession number MK076593) to our primer pairs (refer to Table 1), added a stop codon prior to V1 region and used it as a vector for cloning env/rev cassettes amplified from patient samples via OEC. Addition of stop codon was necessary as it simplified the identification of functional clones for further downstream processing, given colony PCR was not possible as OEC was used to swap a $3.2 \mathrm{~kb}$ fragment with a similar sized env/rev cassette from biological samples. Interestingly, using this pcDNA3.1 delta env backbone gave significantly higher number of colonies compared to colonies observed with either pcDNA3.1_ITR or pcDNA3.1_ITR_CCDB suicide vector (Fig. 4b), presumably due to increased regions of homology upstream to the ENVN and downstream to the ENVA ITR sites in 329_14_B1 $\Delta$ Env. To compare the efficiency of OEC, we then cloned the respective env/rev cassettes from the eight infant samples using the commercially available topoisomerase mediated (TOPO Cloning) and T4 DNA polymerase mediated (Infusion Cloning) cloning kits, and observed significantly higher cloning efficiency, indicating that OEC can be used to efficiently clone difficult-toclone HIV-1 env gene (Fig. 4c)

Furthermore, we attempted to simplify and standardize the OEC protocol into a one-pot (single tube) approach for cloning env/rev cassettes. Using the unpurified PCR amplified env/rev cassettes from a sample that gave negligible non-specific background, we could successfully perform OEC as a onepot platform for cloning env/rev cassettes, though a significantly high number of colonies containing the unwanted primer-dimers as insert were observed. Further titrating the initial concentration of primers used for env/rev amplification did decrease the number of unwanted primer-dimer colonies, but unwanted clones containing primer-dimers could not be completely eliminated. Utilizing touchdown PCR for amplification of env/rev cassettes minimized the number of spurious primer-dimer clones to almost negligible, though it also led to reduced number of colonies after successful OEC. Of particular note, one-pot approach only worked for env/rev cassettes amplified via SGA and not bulk PCR. Amplification of env/rev cassettes with touchdown PCR, followed by one-pot OEC reaction 
using higher amount of vector, led to the most satisfactory results, though for better performance, PCR purification prior to OEC is necessary. Touchdown PCR (TD-PCR) was developed as a simpler solution to address the mispriming and production of non-specific (47), spurious products that often result due to poor melting temperature estimations and improper annealing conditions but in case of env/rev cassette amplification from biological samples, even the most optimized PCR conditions gave non-specific amplification in certain samples. PCRs for env/rev cassettes require optimization for each biological sample but given the extensive diversity and circulating mutant pool in an infected individual, optimized primers, on average, can minimize the requirement for PCR optimization from sample to sample.

Once we successfully swapped patient amplified env/rev cassettes into the HIV-25710_2_42 backbone, another versatility of OEC became apparent. Given that swapping env/rev cassettes from one source into another could easily be achieved via OEC, we next utilized the same procedure to generate chimeras between different env/rev cassettes. Phusion polymerase has been reported to fail PCR amplification when complementary Fw and Rv primers (as suggested for QuikChange Kits) are used for site-directed mutagenesis (48). In our case, we observed persistent positive amplification when using Phusion to perform OEC for site-directed mutagenesis or chimeragenesis, though the primers had to be on average of $60-65$ bp long to achieve more than $90 \%$ efficiency via OEC. For chimeragenesis, primers longer than $25 \mathrm{bp}$ were required for efficiencies $>90 \%$. Though, of note, if Dpnl digestion is optimum, OEC is an all or none approach, thereby, with reaction efficiencies approaching $100 \%$. As noted for several other cloning approaches, and reported previously for OEC, cloning efficiency of OEC in the context of HIV-1 mutagenesis and chimeragenesis was a function of insert length, with considerably lower number of colonies observed for larger inserts (swaps for chimeras) compared to smaller inserts (Fig. 5).

Cloning of HIV-1 env/rev cassettes via OEC is easy to monitor and can be optimized for distinct biological samples with minimum modifications to the final protocol described in this study. For OEC, general rules for primer design apply, but additional focus is required for both the 5 - and 3 - ends with even distribution of pyrimidine and purine bases and either a $\mathrm{G}$ or $\mathrm{C}$ at the terminus. Under suboptimal conditions, OEC significantly generates unwanted clones, and therefore, optimization of reaction conditions is a necessity. Utilizing touchdown PCR further increases the efficiency of OEC for cloning env/rev cassettes as higher annealing temperatures compared with the expected primer $\mathrm{Ta}$ during the initial PCR cycles results in increased PCR product specificity, as spurious primer-template interactions are less stable than the specific ones. Substantial non-specific amplification often necessitates PCR purification prior to OEC, and utilization of TD-PCR further simplifies the OEC workflow. To validate the optimized OEC strategy, HIV-1 env/rev cassettes were amplified from 8 infected infants, of which only, a single sample required modified protocols (varied $\mathrm{Ta}$ ) for successful OEC. The flexibility and high-throughput of OEC allows far greater versatility than the current global standard of TOPO mediated cloning for characterizing HIV-1 envelope glycoprotein. SGA amplified env/rev cassettes could be integrated into the vector by a single PCR, bypassing the need for utilizing expensive cloning kits, or novel enzymes such as T4 DNA polymerase, Taq DNA ligase, 
233 exonucleases and recombinases. The requirement to continuously monitor and optimize OEC is in

234 parallel to any long PCR protocol, and requires moderate level of experience and understanding on

235 the user's part. To summarize, herein, we report OEC as an alternate PCR-based approach for

236 cloning HIV-1 env/rev cassettes, that can yield high-efficiency amplification of HIV-1 envelopes with

237 diverse sequence variability.

\section{$238 \quad$ Materials and Methods}

\section{Materials}

240 Phusion DNA polymerase, dNTPs, Dpnl, DMSO and BSA were purchased from Thermo Scientific. $E$.

241 coli TOP10 (Invitrogen) was used for cloning and competent cells were prepared in-house $\left(10^{6}-10^{7}\right.$

$242 \mathrm{cfu} / \mu \mathrm{g})$. DNA oligonucleotides were synthesized by Eurofins genomics, India. The pcDNA3.1 (+)

243 vector was procured from Invitrogen. The pZM247Fv1 $\triangle$ Env was acquired from NIH AIDS Reagent

244 Program (\#11940). The lab generated pseudovirus 329_14_B1 is described previously(18).

\section{Designing modified primers for env/rev amplification using HIV-1 Alignments}

246 The Indian Clade $\mathrm{C}$ tat and nef sequences were compiled from the Los Alamos National Laboratory

247 HIV-1 Sequence Database using the sequence search interface

248 (https://www.hiv.lanl.gov/components/sequence/HIV/search/search.html), and codon-aligned using

249 the HIV align (https://www.hiv.lanl.gov/content/sequence/VIRALIGN/viralign.html) tool using the HMM-

250 align model with compensating mutation occurring within 5 codons to compensate for frameshift. The

251 MEGA-X and ClustalX2 software tools were used for manually editing the alignments. Schematic

252 plots to represent the sequence diversity, a measure of mismatches in the alignments, were

253 generated using the highlighter tool

254 (https://www.hiv.lanl.gov/content/sequence/HIGHLIGHT/highlighter_top.html) with sequences sorted

255 by similarity and gaps in the alignment treated as characters. Previously described primers were used

256 as anchors to design optimized primers for Indian Clade $C$ sequences. Overlaps comprising of 15, 25,

257 and 35 base pairs [5' to the BamHI site, and 3' to the Apal site in pcDNA3.1(+)] with the primer pairs

258 were manually incorporated using the SnapGene Viewer Program. Primers for site-directed

259 mutagenesis of the pseudovirus 329_14_B1 were designed using Agilent's quikchange primer

260 designing tool. Primers for chimeragenesis were manually designed based on swapping inserts of

261 varying length into the 329_14_B1 HIV-1 pseudoviral clone utilizing SnapGene Viewer Program, with

262 normal primer designing rules of $20-25$ bp length with a $\mathrm{Tm}$ of approximately $60^{\circ} \mathrm{C}$, ideally ending with

$263 \mathrm{G} / \mathrm{C}$ with last 6 bp containing equal number of pyrimidine and purine bases.

\section{Vector design and construction}

265 pcDNA3.1_ITR, pcDNA3.1_ITR_CCDB and pcDNA3.1delta Env were engineered using the

266 mammalian expression vector pcDNA3.1 (+) through a series of overlap extension cloning reactions.

267 pcDNA3.1_ITR was generated via cloning ITR sites ENVA (5` -

268 CACCGGCTTAGGAATTTACTATGGCAGGAAG - 3`) and ENVN (5` -

269 TGCCAATCAGGGAAAAAGCCTTGTGTG - 3`) upstream to BamHI and downstream to Apal 
270 restriction site respectively. Vector pcDNA3.1_ITR_CCDB, CCDB gene was PCR amplified from vector ZM247Fv1 $\triangle$ Env using primers ENVA_CCDB_Forward (5` -

272 TAGGAATTTACTATGGCAGGAAGATGCAGTTTAAGGTTTACACC - 3`) and ENVN_CCDB_Reverse amplicon was next swapped using OEC into $10 \mathrm{ng}$ of vector pCDNA3.1_ITR using CCDB gene as megaprimer at a ratio of $1: 350$. The PCR conditions used were an initial denaturation at $98^{\circ} \mathrm{C}$ for 2 min followed by 16 cycles of denaturation at $98^{\circ} \mathrm{C}$ for $10 \mathrm{sec}$, annealing at $64^{\circ} \mathrm{C}$ for $30 \mathrm{sec}$, and extension at $72^{\circ} \mathrm{C}$ for $3 \mathrm{~min}$ and a final extension at $72^{\circ} \mathrm{C}$ for $5 \mathrm{~min}$. The pcDNA3.1 $\Delta \mathrm{Env}$ (in pseudoviral backbone 329_14_B1) was generated by inserting a stop codon prior to V1 region of the env gene using primers V1_Stop_Forward (5` V1_Stop_Reverse (5' sequencing to confirm successful generation of vectors.

\section{Amplification of env/rev cassettes and overlap extension cloning (OEC) PCR}

The env/rev cassettes of HIV-1 from infected infants were amplified as described previously. Briefly, viral RNA was isolated from $140 \mu$ l of plasma using QIAamp Viral RNA Mini Kit, reverse transcribed, using gene specific primer OFM19 (5` - GCACTCAAGGCAAGCTTTATTGAGGCTTA - 3') and envelope gene using High Fidelity Phusion DNA Polymerase (New England Biolabs). Nested PCRs were performed, using primers given in table 1 with the following PCR conditions; an initial denaturation at $98^{\circ} \mathrm{C}$ for $2 \mathrm{~min}$, followed by 25 cycles of denaturation at $98^{\circ} \mathrm{C}$ for $10 \mathrm{sec}$, annealing at $59^{\circ} \mathrm{C}$ for $30 \mathrm{sec}$, and extension at $72^{\circ} \mathrm{C}$ for $2 \mathrm{~min}$ and a final extension at $72^{\circ} \mathrm{C}$ for $5 \mathrm{~min}$. A second round PCR was performed using first round amplicons with reaction conditions of initial denaturation of $98^{\circ} \mathrm{C}$ for $2 \mathrm{~min}$, followed by 35 cycles of denaturation at $98^{\circ} \mathrm{C}$, annealing at $59^{\circ} \mathrm{C}$ for $30 \mathrm{sec}$, and extension at $72^{\circ} \mathrm{C}$ for $90 \mathrm{sec}$, and a final extension at $72^{\circ} \mathrm{C}$ for $90 \mathrm{secs}$. The env/rev cassettes were PCR purified using the QIAquick PCR \& Gel Cleanup Kit and utilized as megaprimers for overlap extension cloning into pcDNA3.1 vector backbone (pcDNA3.1_ITR, pcDNA3.1_ITR_CCDB, and pcDNA3.1 $\triangle \mathrm{Env}$ ). Overlap extension cloning was performed with varying amount of pcDNA vector backbone and env/rev cassettes with PCR reaction conditions of initial denaturation at $98^{\circ} \mathrm{C}$ for $2 \mathrm{~min}$, followed by $10-24$ cycles of denaturation at $98^{\circ} \mathrm{C}$ for $10 \mathrm{sec}$, annealing at $62^{\circ} \mathrm{C}$ for $30 \mathrm{sec}$, and extension at $72^{\circ} \mathrm{C}$ for $4.5 \mathrm{~min}$, and a final extension of $5 \mathrm{~min}$ at $72^{\circ} \mathrm{C}$. In addition, $1 \%$ DMSO and $1 \%$ BSA ( $1 \mathrm{mg} / \mathrm{ml}$ ) were used as additives for OEC of env/rev cassettes. The PCR products were analyzed using $0.8 \%$ Agarose gel electrophoresis.

\section{Transformation and DNA Sequencing}

306 Aliquots $(5 \mu \mathrm{l})$ of undigested and Dpnl digested OEC PCR amplicons were directly transformed into 307 in-house generated chemically competent TOP10 cells. Cells were spread on 2XYT-agar plates 
containing $100 \mu \mathrm{g} / \mathrm{ml}$ of ampicillin sodium salt and incubated overnight at $30^{\circ} \mathrm{C}$. For env $/ \mathrm{rev}$ cassettes,

incubation at lower temperature reduced the toxicity associated with problematic DNA sequences (42-44). The number of colonies grown on each plate were calculated and normalized to per $\mathrm{ng}$ of starting vector backbone to calculate cloning efficiency (colony forming units per ng of DNA). DNA Sequencing was performed commercially from eurofins genomics, India.

\section{Acknowledgments}

314 We are thankful to all the study subjects for participating in this study and the NIH AIDS Reagent 315 program for providing HIV-1 envelope pseudovirus plasmids.

\section{Funding}

317 This work was funded by Department of Biotechnology, India (BT/PR5066/MED/1582/2012 and

318 BT/PR30120/MED/29/1339/2018) and Science and Engineering Research Board, Department of

319 Science and Technology, India (EMR/2015/001276). The Junior Research Fellowship (January 2016

320 - December 2018) and Senior Research Fellowship (January 2019 - October 2019) to N.M was

321 supported by University Grants Commission (UGC), India.

\section{Author Contributions}

323 N.M, S.S and K.L designed the study. N.M, A.D and S.S performed the experimental work. N.M

324 analyzed the data, wrote the initial manuscript, revised and finalized the manuscript. K.L wrote,

325 edited, revised and finalized the manuscript.

\section{Competing Interests}

327 The authors declare no competing financial interests.

\section{Data and Material Availability}

All data required to state the conclusions in the paper are present in the paper and/or the supplementary data. Additional information related to the paper, if required, can be requested from the authors.

\section{References}

1. Korber B, Hraber P, Wagh K, Hahn BH. Polyvalent vaccine approaches to combat HIV-1 diversity. Immunol Rev. 2017;275(1):230-44.

2. Ackerman ME, Barouch DH, Alter G. Systems serology for evaluation of HIV vaccine trials. Immunol Rev. 2017;275(1):262-70.

3. Bhiman JN, Anthony C, Doria-Rose NA, Karimanzira O, Schramm CA, Khoza T, et al. Viral variants that initiate and drive maturation of V1V2-directed HIV-1 broadly neutralizing antibodies. Nat Med. 2015 Nov;21(11):1332-6.

4. Wibmer CK, Bhiman JN, Gray ES, Tumba N, Abdool Karim SS, Williamson C, et al. Viral escape from HIV-1 neutralizing antibodies drives increased plasma neutralization breadth through sequential recognition of multiple epitopes and immunotypes. PLoS Pathog. 2013 Oct;9(10):e1003738. 
5. Doria-Rose NA, Schramm CA, Gorman J, Moore PL, Bhiman JN, DeKosky BJ, et al. Developmental pathway for potent V1V2-directed HIV-neutralizing antibodies. Nature. 2014 May $1 ; 509(7498): 55-62$.

6. Kwong PD, Mascola JR. HIV-1 Vaccines Based on Antibody Identification, B Cell Ontogeny, and Epitope Structure. Immunity. 2018 15;48(5):855-71.

7. Sok D, Burton DR. Recent progress in broadly neutralizing antibodies to HIV. Nat Immunol. 2018 Nov;19(11):1179-88.

8. Sanders RW, Derking R, Cupo A, Julien J-P, Yasmeen A, de Val N, et al. A next-generation cleaved, soluble HIV-1 Env trimer, BG505 SOSIP.664 gp140, expresses multiple epitopes for broadly neutralizing but not non-neutralizing antibodies. PLoS Pathog. 2013 Sep;9(9):e1003618.

9. Sanders RW, van Gils MJ, Derking R, Sok D, Ketas TJ, Burger JA, et al. HIV-1 VACCINES. HIV-1 neutralizing antibodies induced by native-like envelope trimers. Science. $2015 \mathrm{Jul}$ 10;349(6244):aac4223.

10. Pugach P, Ozorowski G, Cupo A, Ringe R, Yasmeen A, de Val N, et al. A native-like SOSIP.664 trimer based on an HIV-1 subtype B env gene. J Virol. 2015 Mar;89(6):3380-95.

11. Klasse PJ, Ketas TJ, Cottrell CA, Ozorowski G, Debnath G, Camara D, et al. Epitopes for neutralizing antibodies induced by HIV-1 envelope glycoprotein BG505 SOSIP trimers in rabbits and macaques. PLoS Pathog. 2018;14(2):e1006913.

12. Sliepen K, Han BW, Bontjer I, Mooij P, Garces F, Behrens A-J, et al. Structure and immunogenicity of a stabilized HIV-1 envelope trimer based on a group-M consensus sequence. Nat Commun. 2019 29;10(1):2355.

13. Dingens AS, Arenz D, Weight H, Overbaugh J, Bloom JD. An Antigenic Atlas of HIV-1 Escape from Broadly Neutralizing Antibodies Distinguishes Functional and Structural Epitopes. Immunity. 2019 19;50(2):520-532.e3.

14. Song H, Hora B, Giorgi EE, Kumar A, Cai F, Bhattacharya T, et al. Transmission of Multiple HIV-1 Subtype C Transmitted/founder Viruses into the Same Recipients Was not Determined by Modest Phenotypic Differences. Sci Rep. 2016 02;6:38130.

15. Li M, Gao F, Mascola JR, Stamatatos L, Polonis VR, Koutsoukos M, et al. Human immunodeficiency virus type 1 env clones from acute and early subtype $B$ infections for standardized assessments of vaccine-elicited neutralizing antibodies. J Virol. 2005 Aug;79(16):10108-25.

16. Li M, Salazar-Gonzalez JF, Derdeyn CA, Morris L, Williamson C, Robinson JE, et al. Genetic and neutralization properties of subtype $C$ human immunodeficiency virus type 1 molecular env clones from acute and early heterosexually acquired infections in Southern Africa. J Virol. 2006 Dec;80(23):11776-90.

17. Kulkarni SS, Lapedes A, Tang H, Gnanakaran S, Daniels MG, Zhang M, et al. Highly complex neutralization determinants on a monophyletic lineage of newly transmitted subtype C HIV-1 Env clones from India. Virology. 2009 Mar 15;385(2):505-20.

18. Mishra N, Makhdoomi MA, Sharma S, Kumar S, Dobhal A, Kumar D, et al. Viral Characteristics Associated with Maintenance of Elite Neutralizing Activity in Chronically HIV-1 Clade C-Infected Monozygotic Pediatric Twins. J Virol. 2019 Sep 1;93(17).

19. Keele BF, Giorgi EE, Salazar-Gonzalez JF, Decker JM, Pham KT, Salazar MG, et al. Identification and characterization of transmitted and early founder virus envelopes in primary HIV-1 infection. Proc Natl Acad Sci USA. 2008 May 27;105(21):7552-7. 
20. Kouyos RD, Rusert P, Kadelka C, Huber M, Marzel A, Ebner H, et al. Tracing HIV-1 strains that imprint broadly neutralizing antibody responses. Nature. 2018 Sep;561(7723):406-10.

21. MacLeod DT, Choi NM, Briney B, Garces F, Ver LS, Landais E, et al. Early Antibody Lineage Diversification and Independent Limb Maturation Lead to Broad HIV-1 Neutralization Targeting the Env High-Mannose Patch. Immunity. 2016 17;44(5):1215-26.

22. Bar KJ, Li H, Chamberland A, Tremblay C, Routy JP, Grayson T, et al. Wide variation in the multiplicity of HIV-1 infection among injection drug users. J Virol. 2010 Jun;84(12):6241-7.

23. Mabvakure BM, Scheepers C, Garrett N, Abdool Karim S, Williamson C, Morris L, et al. Positive Selection at Key Residues in the HIV Envelope Distinguishes Broad and Strain-Specific Plasma Neutralizing Antibodies. J Virol. 2019 15;93(6).

24. Kijak GH, Sanders-Buell E, Chenine A-L, Eller MA, Goonetilleke N, Thomas R, et al. Rare HIV-1 transmitted/founder lineages identified by deep viral sequencing contribute to rapid shifts in dominant quasispecies during acute and early infection. PLoS Pathog. 2017 Jul;13(7):e1006510.

25. Kijak GH, Sanders-Buell E, Pham P, Harbolick EA, Oropeza C, O'Sullivan AM, et al. Nextgeneration sequencing of HIV-1 single genome amplicons. Biomol Detect Quantif. 2019 Mar;17:100080.

26. Bryksin AV, Matsumura I. Overlap extension PCR cloning: a simple and reliable way to create recombinant plasmids. BioTechniques. 2010 Jun;48(6):463-5.

27. Bryksin A, Matsumura I. Overlap extension PCR cloning. Methods Mol Biol. 2013;1073:31-42.

28. Zhang H, Liu C-J, Jiang H, Zhou L, Li W-Y, Zhu L-Y, et al. Mega primer-mediated molecular cloning strategy for chimaeragenesis and long DNA fragment insertion. Biosci Rep. 2017 30;37(2).

29. Guo W, Xie B, Jiang M, Zhu X-J, Qiu M, Dai Z-M. An improved overlap extension PCR for simultaneous multiple sites large fragments insertion, deletion and substitution. Sci Rep. 2019 Oct 30;9(1):15637.

30. Huang J, Yu Z, Li M-H, Li N, Zhou J, Zheng Y-G. A strategy for seamless cloning of large DNA fragments from Streptomyces. BioTechniques. 2015 Oct;59(4):193-4, 196, 198-200.

31. Liu C-J, Jiang H, Wu L, Zhu L-Y, Meng E, Zhang D-Y. OEPR Cloning: an Efficient and Seamless Cloning Strategy for Large- and Multi-Fragments. Sci Rep. 2017 16;7:44648.

32. Motohashi K. Seamless Ligation Cloning Extract (SLiCE) Method Using Cell Lysates from Laboratory Escherichia coli Strains and its Application to SLiP Site-Directed Mutagenesis. Methods Mol Biol. 2017;1498:349-57.

33. Zhong C, You C, Wei P, Zhang Y-HP. Simple Cloning by Prolonged Overlap Extension-PCR with Application to the Preparation of Large-Size Random Gene Mutagenesis Library in Escherichia coli. Methods Mol Biol. 2017;1472:49-61.

34. Chaudhary VK, Shrivastava N, Verma V, Das S, Kaur C, Grover P, et al. Rapid restriction enzyme-free cloning of PCR products: a high-throughput method applicable for library construction. PLoS ONE. 2014;9(10):e111538.

35. Li C, Wen A, Shen B, Lu J, Huang Y, Chang Y. FastCloning: a highly simplified, purification-free, sequence- and ligation-independent PCR cloning method. BMC Biotechnol. 2011 Oct 12;11:92.

36. Li MZ, Elledge SJ. Harnessing homologous recombination in vitro to generate recombinant DNA via SLIC. Nat Methods. 2007 Mar;4(3):251-6. 
431 37. Zhou MY, Gomez-Sanchez CE. Universal TA cloning. Curr Issues Mol Biol. 2000 Jan;2(1):1-7.

432 38. Zhang Y, Werling U, Edelmann W. SLiCE: a novel bacterial cell extract-based DNA cloning

39. García-Nafría J, Watson JF, Greger IH. IVA cloning: A single-tube universal cloning system exploiting bacterial In Vivo Assembly. Sci Rep. 2016 06;6:27459.

40. Beyer HM, Gonschorek P, Samodelov SL, Meier M, Weber W, Zurbriggen MD. AQUA Cloning: A Versatile and Simple Enzyme-Free Cloning Approach. PLoS ONE. 2015;10(9):e0137652.

41. Quan J, Tian J. Circular polymerase extension cloning. Methods Mol Biol. 2014;1116:103-17.

42. van der Kuyl AC, Berkhout B. The biased nucleotide composition of the HIV genome: a constant factor in a highly variable virus. Retrovirology. 2012 Nov 6;9:92.

43. van Hemert F, van der Kuyl AC, Berkhout $B$. On the nucleotide composition and structure of retroviral RNA genomes. Virus Res. 2014 Nov 26;193:16-23.

44. Benureau Y, Colin P, Staropoli I, Gonzalez N, Garcia-Perez J, Alcami J, et al. Guidelines for cloning, expression, purification and functional characterization of primary HIV-1 envelope glycoproteins. J Virol Methods. 2016;236:184-95.

45. Mishra N, Sharma S, Dobhal A, Kumar S, Chawla H, Singh R, et al. Broadly neutralizing plasma antibodies effective against diverse autologous circulating viruses in infants with multivariant HIV-1 infection. bioRxiv. 2020 Feb 3;837039.

46. Tripathi A, Swaroop S, Varadarajan R. Molecular Determinants of Temperature-Sensitive Phenotypes. Biochemistry. 2019 02;58(13):1738-50.

47. Korbie DJ, Mattick JS. Touchdown PCR for increased specificity and sensitivity in PCR amplification. Nat Protoc. 2008;3(9):1452-6.

48. Xia Y, Chu W, Qi Q, Xun L. New insights into the QuikChange ${ }^{T M}$ process guide the use of Phusion DNA polymerase for site-directed mutagenesis. Nucleic Acids Res. 2015 Jan;43(2):e12. 


\section{$458 \quad$ Figures and Tables}

459

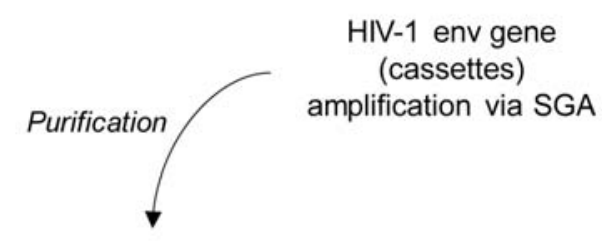

Utilization of env cassettes as megaprimer for OEC

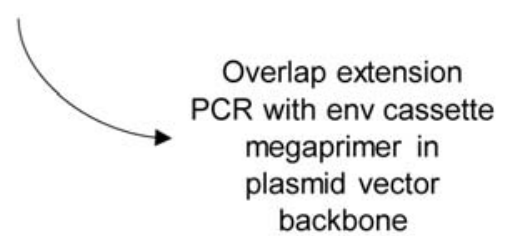

Transformation into competent E.coli

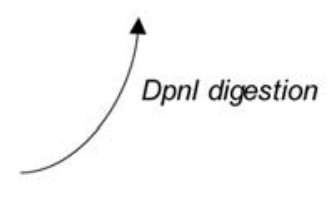

460 Figure 1 - Schematic flow for overlap extension cloning. Outline of the major steps involved in

461 OEC is provided. The key step outlining the utilization of env/rev cassettes as megaprimer is boxed.

462 First, env/rev cassettes are PCR amplified from HIV-1 infected individuals and then used as

463 megaprimers in plasmid vectors containing the integration sites (sequence of forward and reverse

464 primer for env/rev cassette amplification) (see the vector map provided in supplementary figure 2),

465 ultimately leading to generation of double nicked plasmid which is transformed into E.coli. 


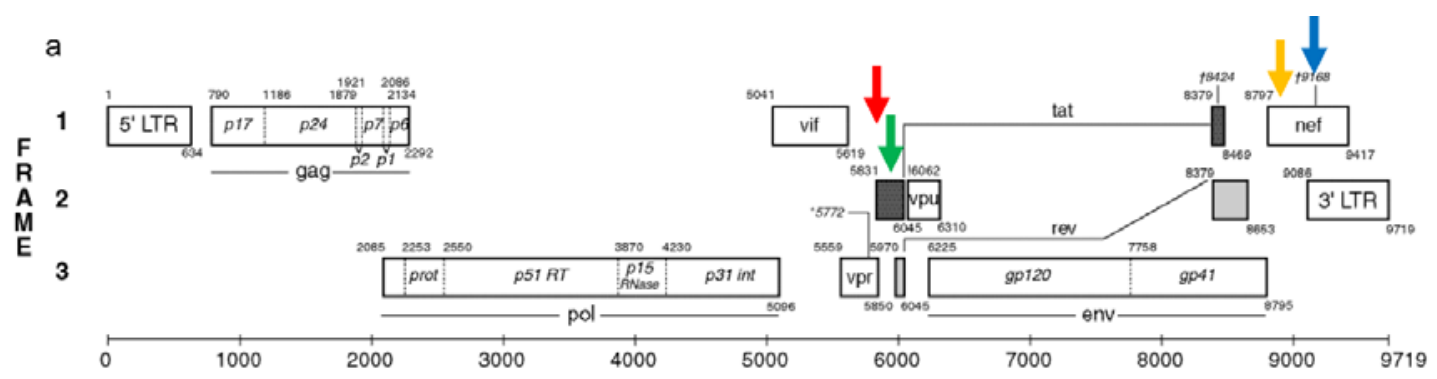

b

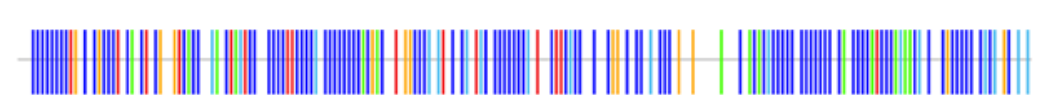

C

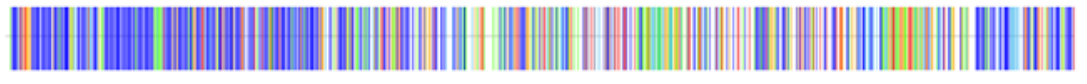

d

d

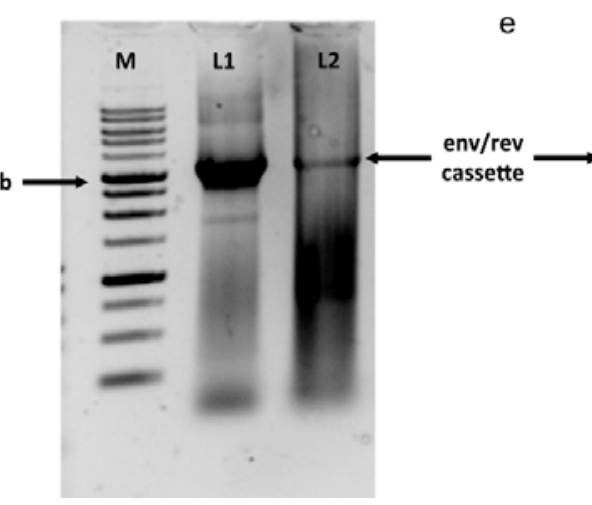

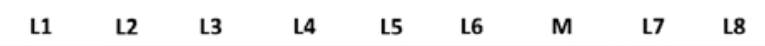

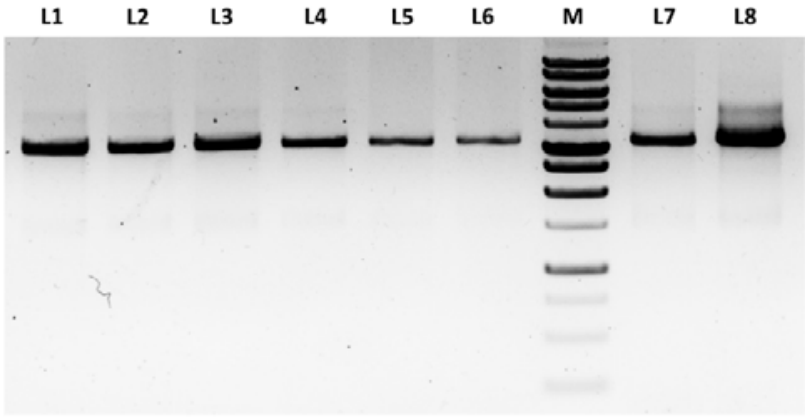

Figure 2 - Optimizing the amplification of env/rev cassettes. (a) Schematic map of the HIV-1 genome showing the position of nested PCR primers. Within the first exon of tat, 1st round forward primer (Fw1) is marked by red, 2nd round forward primer (Fw2) is marked by green, and within the nef region 1st round reverse primer (Rv1) is marked by blue and 2nd round reverse primer (Rv2) is marked by yellow arrow. $(b-c)$ Schematic map of the tat and nef region representing the sequence diversity in Indian clade C sequences reported in LANL HIV-1 database. Dark blue lines represent position that contain any of the four bases, while green lines represent adenine, red line represent thymine, orange line represent guanine and sky-blue lines represent cytosine relative to the Indian clade $C$ consensus sequence generated by aligning available sequence from HIV-1 database. $(d-e)$ Gel pictures showing amplified env/rev cassettes $(\sim 3.2 \mathrm{~kb})$ from updated primer pairs as mentioned in table 1. In d, L1 represent plasmid control (HIV-25710, NIH AIDS Reagent Program \#11505) while L2 represents amplified env/rev cassette using multiplex approach while in e, L1 to L8 represent env/rev amplification after optimization of primer binding using iterative selection approach. 


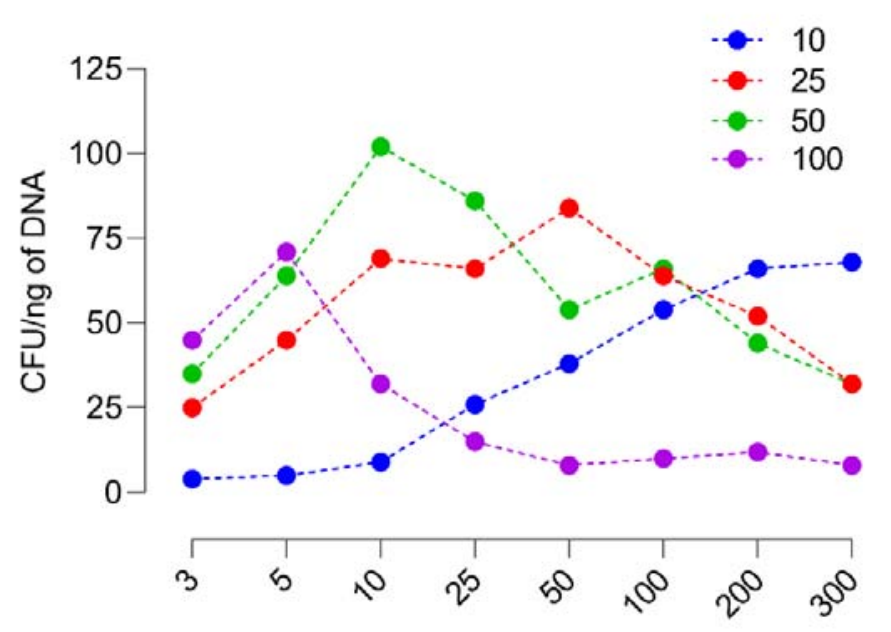

480

481 Figure 3 - High concentration of megaprimer inhibits OEC. Overlap extension cloning of env/rev

482 cassette from a single infant was cloned in pcDNA3.1_ITR at increasing ratios of vector to insert (1:3

483 to $1: 300$ ) as well as increasing amount of starting vector (10 to $100 \mathrm{ng}$ of pcDNA3.1_ITR). Colony

484 forming unit (CFU) were counted as the number of colonies normalized to starting vector amount. A

485 vector to insert ratio of 1: 10 to 1:20 with starting vector amount of $10 \mathrm{ng}$ showed maximum cloning

486 efficiency across samples. 


\section{a}

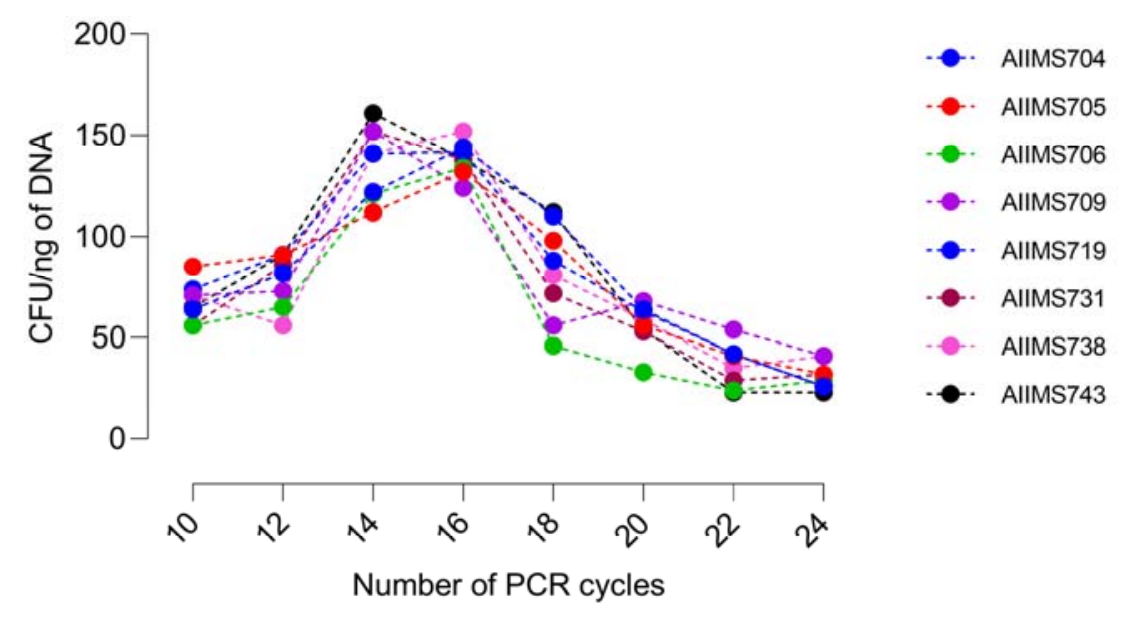

b

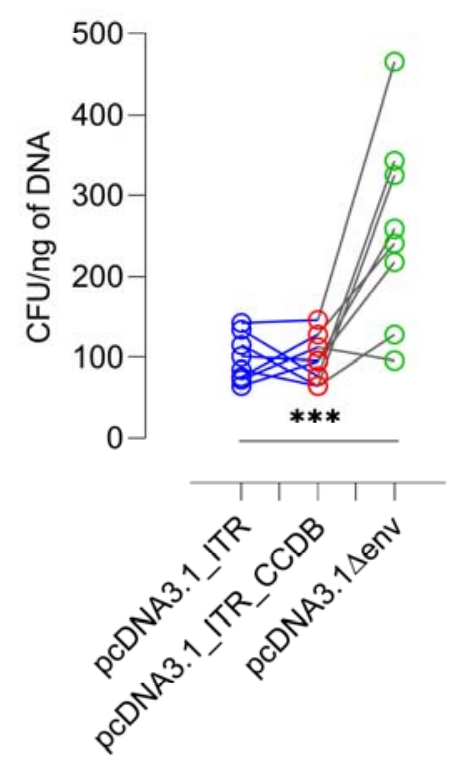

C
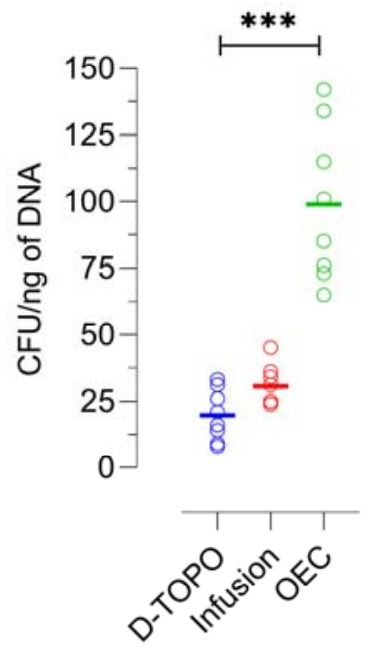

488 Figure 4-OEC efficiency as a function of amplification cycle. Overlap extension cloning

489 performed for env/rev cassettes amplified from eight infant plasma samples was iteratively optimized

490 as a function of amplification cycles in OEC PCR. 14 - 16 cycles consistent gave satisfactory cloning

491 efficiency across samples. Colony forming unit (CFU) were counted as the number of colonies

492 normalized to starting vector amount. (b) Diverse vector backbones were utilized to further improve

493 the OEC cloning efficiency. Using pcDNA3.1 1 env vector backbone, maximum efficiency of OEC was

494 achieved. (c) Comparison of the cloning efficiency of the topoisomerase mediated (D-TOPO), T4 DNA

495 polymerase mediated (Infusion) and overlap extension PCR mediated (OEC) cloning strategies for

496 cloning the env/rev cassettes from eight HIV-1 infected infants. 


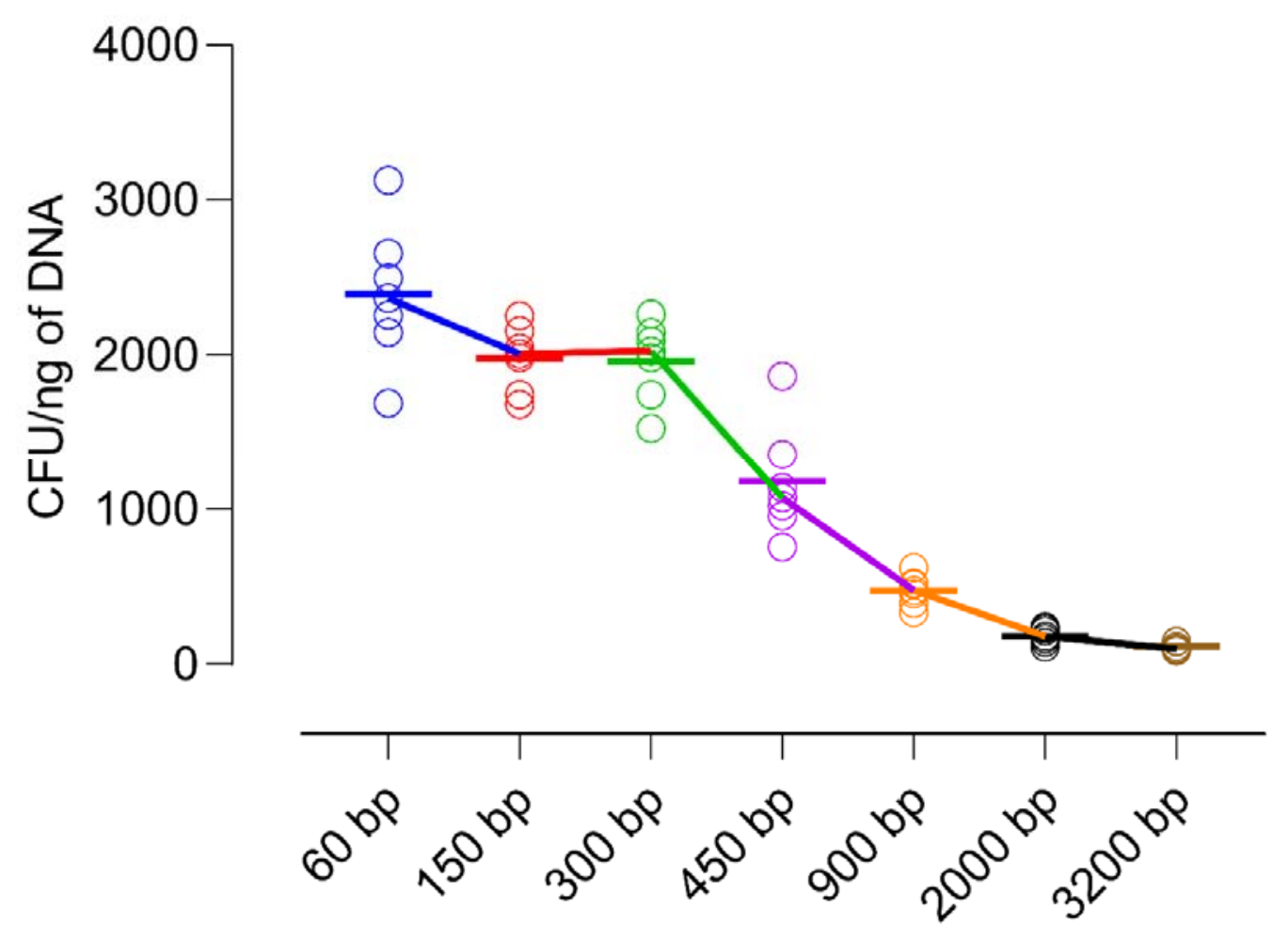

Figure 5 - Efficiency of Overlap Extension Cloning for chimeragenesis in env/rev cassettes.

499 With varying length of megaprimers (inserts to be swapped), Overlap extension cloning was

500 performed for chimeragenesis. Results are reported for six independent experiments. Colony forming

501 unit (CFU) were counted as the number of colonies normalized to starting vector amount. All the

502 megaprimers were swapped into the vector backbone pcDNA3.1-329_14_B1. Marked reduction in

503 cloning efficiency (chimeragenesis) with increasing length of insert was observed though satisfactory 504 results were still observed.

\begin{tabular}{|l|l|l|l|}
\hline $\begin{array}{l}\text { Nested PCR } \\
\text { Round }\end{array}$ & Primer ID & Sequence $\left(5^{`}-3^{`}\right)$ & $\begin{array}{l}\text { HXB2 } \\
\text { Position }\end{array}$ \\
\hline 1st Round & Fw1.1_VIF1 & TAGAGCCCTGGAATCATCCAGGAAG & $5852-5876$ \\
\hline 1st Round & Fw1.2_VIF1 & TAGAGCCCTGGAATCATCCAGGCAG & $5852-5876$ \\
\hline 1st Round & Fw1.3_VIF1 & TAGAGCCCTGGAACCATCCAGGAAG & $5852-5876$ \\
\hline 1st Round & Fw1.4_VIF1 & TAGAGCCCTGGAACCATCCAGGCAG & $5852-5876$ \\
\hline 1st Round & Rv1.1_OFM19 & GCACTCAAGGCAAGCTTTATTGAGGCTTA & $9604-9632$ \\
\hline 1st Round & Rv1.2_OFM19 & GCACTCAAGGCAAGCCTTATTGAGGCTTA & $9604-9632$ \\
\hline 2nd Round & Fw2.1_ENVA & GGCTTAGGCATTTACTATGGCAGGAAG & $5951-5980$ \\
\hline 2nd Round & Fw2.2_ENVA & GGCTTAGGAATTTCCTATGGCAGGAAG & $5951-5980$ \\
\hline 2nd Round & Fw2.3_ENVA & GGCTTAGGTATTTACTATGGCAGGAAG & $5951-5980$ \\
\hline 2nd Round & Fw2.4_ENVA & GGCTTAGGCATTTCCTATGGCAGGAAG & $5951-5980$ \\
\hline
\end{tabular}




\begin{tabular}{|l|l|l|l|}
\hline 2nd Round & Fw2.5_ENVA & GGCTTAGGAATTTACTATGGCAGGAAG & $5951-5980$ \\
\hline 2nd Round & Fw2.6_ENVA & GGCTTAGGTATTTCCTATGGCAGGAAG & $5951-5980$ \\
\hline 2nd Round & Fw2.7_ENVA & GGCTTAAGCATTTACTATGGCAGGAAG & $5951-5980$ \\
\hline 2nd Round & Fw2.8_ENVA & GGCTTAAGAATTTCCTATGGCAGGAAG & $5951-5980$ \\
\hline 2nd Round & Fw2.9_ENVA & GGCTTAAGTATTTACTATGGCAGGAAG & $5951-5980$ \\
\hline 2nd Round & Fw2.10_ENVA & GGCTTAAGCATTTCCTATGGCAGGAAG & $5951-5980$ \\
\hline 2nd Round & Fw2.11_ENVA & GGCTTAAGAATTTACTATGGCAGGAAG & $5951-5980$ \\
\hline 2nd Round & Fw2.12_ENVA & GGCTTAAGTATTTCCTATGGCAGGAAG & $5951-5980$ \\
\hline 2nd Round & Rv2.1_ENVN & TGCCAATCAGGGAAAAAGCCTTGTGTG & $9144-9172$ \\
\hline 2nd Round & Rv2.2_ENVN & TGCCAATCAGGGAAATAGCCTTGTGTG & $9144-9172$ \\
\hline 2nd Round & Rv2.3_ENVN & TGCCAATCAGGGAAACAGCCTTGTGTG & $9144-9172$ \\
\hline 2nd Round & Rv2.4_ENVN & TGCCAATCAGGGAAGAAGCCTTGTGTG & $9144-9172$ \\
\hline 2nd Round & Rv2.5_ENVN & TGCCAATCAGGGAAGTAGCCTTGTGTG & $9144-9172$ \\
\hline 2nd Round & Rv2.6_ENVN & TGCCAATCAGGGAAGCAGCCTTGTGTG & $9144-9172$ \\
\hline
\end{tabular}

506 pair Fw1.3_VIF1/Rv1.1_OFM19 for $1^{\text {st }}$ round PCR and Fw2.6_ENVA/Rv2.4_ENVN for $2^{\text {nd }}$ round gave 507 most satisfactory env/rev cassette amplification across samples.

508 Supplementary Figures

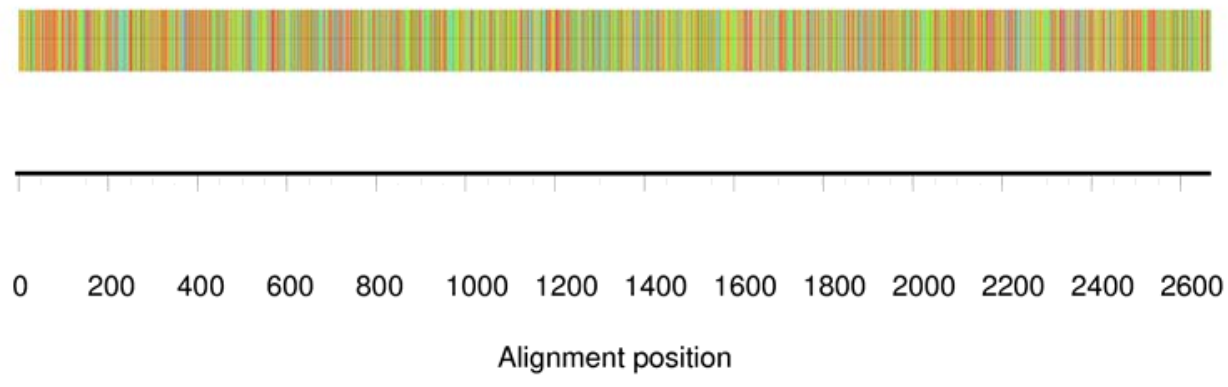

Supplementary Figure 1 - Unusual base composition of HIV-1 env gene. HIV-1 contains an

511 above average percentage of adenine (A) nucleotides, while cysteine (C) nucleotides are extremely

512 low as evident by the large percentage of red (adenine), and limited percentage of blue (cytosine)

513 lines in the base composition plot for consensus env sequence (HIV-1_env) generated by aligning the

514 env sequences of Indian clade C available in LANL HIV-1 database

515 https://www.hiv.lanl.gov/components/sequence/HIV/search/search.html). 


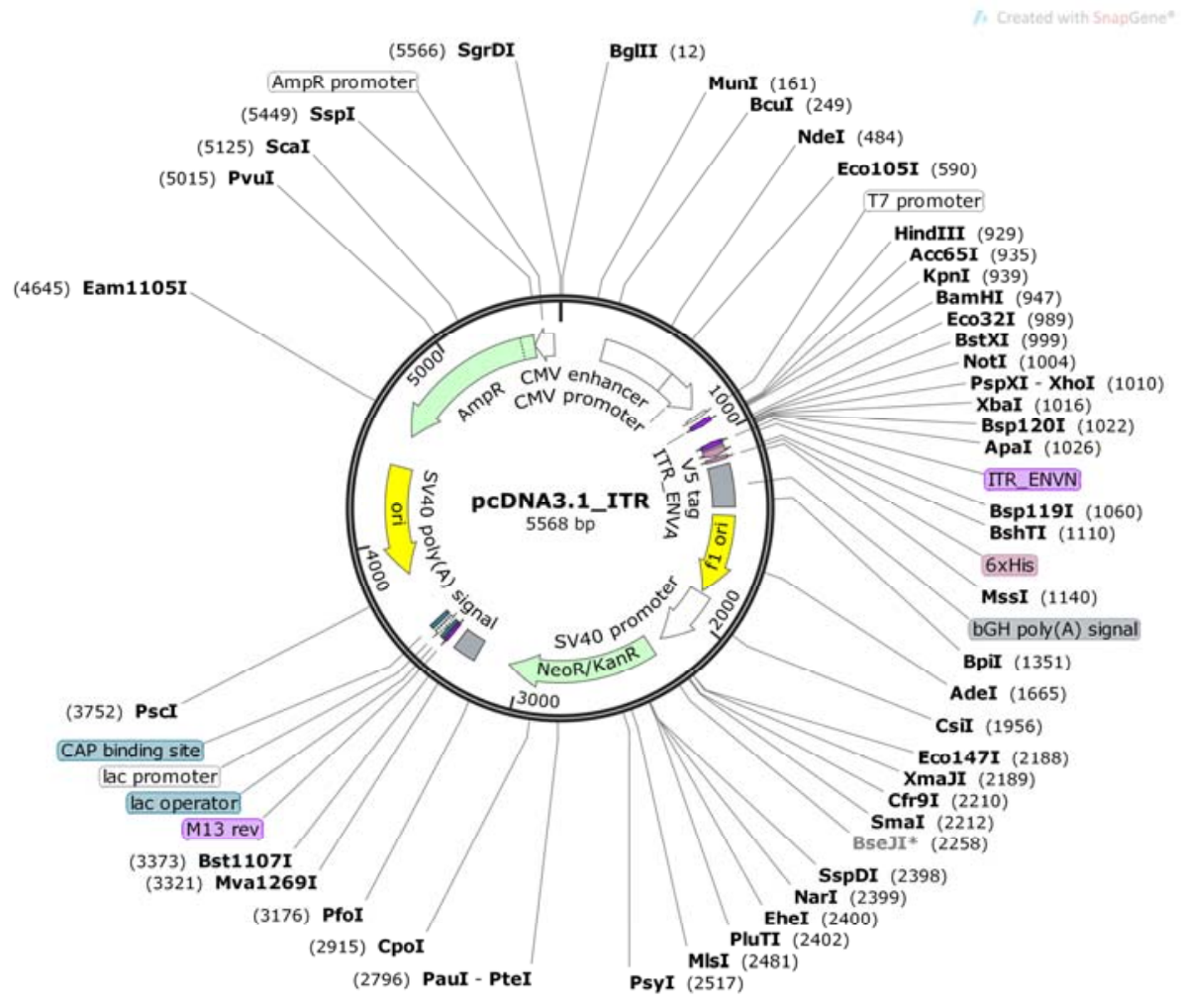

517 Supplementary Figure 2 - Vector map of modified pcDNA3.1 plasmid utilized for cloning HIV-1

518 env/rev cassettes. In pcDNA3.1 (+) backbone, ENVA (Fw2) and ENVN (Rv2) sites optimized based

519 on results of figure 1 were integrated (ITR_ENVA and ITR_ENVN) into pcDNA3.1 by performing two

520 rounds of overlap extension PCR upstream to HindIII and downstream to Apal restriction sites. 